\title{
Velocity-selected magnetic guiding of Zeeman-decelerated hydrogen atoms
}

\author{
Katrin Dulitz ${ }^{1, a}$ and Timothy P. Softley ${ }^{1, b}$ \\ Department of Chemistry, University of Oxford, Chemistry Research Laboratory, 12 Mansfield Road, Oxford, OX1 3TA, UK
}

Received 3rd August 2015 / Received in final form 1st November 2015

Published online 26 January 2016

(c) The Author(s) 2016. This article is published with open access at Springerlink.com

\begin{abstract}
An original design of magnetic guide is presented, suitable for use with Zeeman-decelerated supersonic beams of ground-state hydrogen atoms and other light paramagnetic species. Three-dimensional particle trajectory simulations show that, by combining a series of permanent-magnet Halbach arrays with pulsed high-current wire electromagnets, this guide can be used to efficiently transmit the slow, decelerated atoms and discard the faster, undecelerated atoms and other species in the gas beam. The curved guide would be suitable for guiding hydrogen atoms into an ion trap to investigate low temperature ion-molecule collisions. It is also shown that the device could be used for the guiding or velocity selection from an undecelerated supersonic or effusive beam.
\end{abstract}

\section{Introduction}

The study of cold and ultracold collisions paves the way towards the understanding of chemical reaction dynamics in a still vastly unexplored temperature regime. At subKelvin temperatures, where the de Broglie wavelength becomes comparable to or larger than the range of the interparticle interactions, the chemical reactivity is dominated by non-classical effects such as tunnelling, non-classical reflections and resonances [1,2]. Recently, quantum effects in chemical reactions have also been observed experimentally [3-6]. Since only very few quantum states are involved in a low-temperature collision, thermal averaging is avoided, which allows for an accurate interpretation of experimental data and - through the possibility to calculate improved potential energy surfaces - will also lead to a better understanding of collision processes at higher temperatures.

The possibility to manipulate and control supersonic beams using external fields has enabled the study of elastic, inelastic and reactive collisions in crossed and mergedbeam configurations at a previously unattained level of accuracy and/or in temperature regimes that can typically not be reached with established methods $[7,8]$. In addition to that, supersonic beam decelerators or other slowing techniques make it possible to perform crossed-beam scattering studies as a function of collision energy using a fixed experimental geometry. The study of chemical reactions involving open-shell (free-radical) systems, e.g. using a Zeeman decelerator, is interesting in particular, because

\footnotetext{
a Present address: Laboratory of Physical Chemistry, ETH Zürich, Vladimir-Prelog-Weg 2, 8093 Zürich, Switzerland

b e-mail: tim.softley@chem.ox.ac.uk
}

very little is known about these reactions at low collision energies. Our own interest is in studying the collisions of radicals with molecular ions for which there is a vast gap in knowledge about reaction rates and their temperature dependence in the low-temperature regime [9].

Zeeman deceleration is an experimental technique relying on the fast switching of magnetic fields within an array of solenoid coils in order to manipulate the translational motion of atomic and molecular systems with a permanent magnetic dipole moment. It can be used to produce mK-cold, velocity-tunable supersonic beams of particles in specific internal quantum states. To study cold ion-radical processes as a function of collision energy, the Zeeman decelerator could be combined with a radiofrequency ion trap, which provides a suitable "reaction vessel" in which laser-cooled Coulomb-crystallised ions can be stored for several hours at a time. To determine chemical reaction rates between the Zeeman-decelerated atoms or molecules and the trapped ions as a function of collision energy, it would be necessary to filter out the precursor molecules, carrier gas atoms and all other particles that are outside the phase-space volume accepted by the decelerator. For example, in experiments with ground-state $\mathrm{H}$ atoms, $\mathrm{NH}_{3}$ precursor molecules, $\mathrm{NH}_{2}$ molecules produced during photolysis, undecelerated and partially decelerated/accelerated $\mathrm{H}$ atoms as well as carrier gas atoms can be transmitted through the Zeeman decelerator and can potentially corrupt or compromise accurate reaction rate measurements.

For a sufficiently long Zeeman decelerator, where the flight times of the decelerated and undecelerated beams are different by at least $500 \mu \mathrm{s}$, a fast-opening mechanical shutter $[10,11]$ or a mechanical chopper wheel [12] 


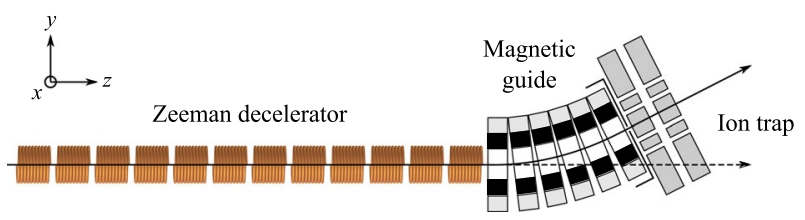

Fig. 1. Schematic representation of a combined Zeeman decelerator-ion trap experiment including a bent magnetic guide; not to scale. A mu-metal cup may be required to shield the trapped ions from stray magnetic fields (indicated aperture between ion trap and guide).

may be used to isolate the decelerated particles from the undecelerated part of the beam. If the different velocity components are not well-separated after deceleration, as in the case of a short 12-stage Zeeman decelerator for $\mathrm{H}$ atoms [13,14], a bent magnetic guide represents another possible way forward. It would both block the precursor and carrier gas particles and act as a low-pass velocity filter for the decelerated particles. A possible setup for a combined Zeeman decelerator-ion trap experiment is illustrated in Figure 1.

An expression for the maximum guidable beam velocity in a curved guide, $v_{z, m}$, can be obtained by setting the transverse magnetic force equal to the centripetal force,

$$
v_{z, m}=\sqrt{\frac{\mu R}{m} \frac{d B(r)}{d r}},
$$

where $R$ is the bend radius and $d B(r) / d r$ is the magnetic field gradient in the transverse direction. Equation (1) shows that, for a given atom or molecule with a magneticmoment-to-mass ratio $\mu / m$, the maximum guidable velocity can be varied by either changing the bend radius or the transverse magnetic field gradient. The first option is not practical for a decelerator-ion-trap arrangement, as it would require a gradual change in the ion trap position and laser alignment to ensure that the guided beam is aimed at the centre of the ion trap (Fig. 1). A magnetic guide made from current-carrying wires does allow for a change in the transverse magnetic field gradient, but this change is too small to enable the manipulation of beams in the supersonic flow regime unless very high currents are used. On the other hand, permanent magnets, e.g. in a hexapole configuration, provide large magnetic field gradients but the magnetic field is not tunable.

There have been various approaches to achieve magnetic guiding, either using wire geometries [4,15-20] or permanent-magnet assemblies [21-26]. However, these designs did not aim at a variation of the maximum guidable velocity which is required for the use of Zeeman-decelerated supersonic beams (typical velocities between $100-500 \mathrm{~m} / \mathrm{s}$ in the case of $\mathrm{H}$ atoms $[13,14])$ in collision-energy-dependent reaction studies in ion traps.

Recently, we were involved in a proposal for a synchrotron for $\mathrm{H}$ atoms based on hybrid magnetic lenses made of both permanent magnets and current-carrying wires in order to study cold collisions between stored $\mathrm{H}$ atoms and supersonic molecular beams [27]. In the following, we propose a scheme for a bent hybrid magnetic
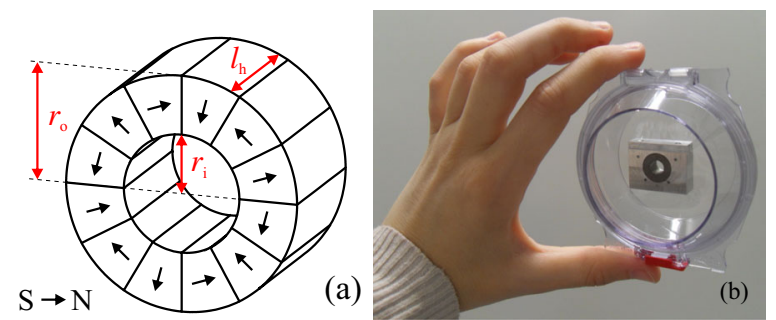

Fig. 2. (a) Schematic drawing of a Halbach array in hexapole configuration. (b) Photograph of a mounted Halbach array built for magnetic guiding and to study particle focusing into a Zeeman decelerator. The dimensions of the Halbach array and its aluminium housing are matched to the size of the deceleration coils and the coil blocks in our Zeeman decelerator [14], respectively.

guide which allows for both high magnetic field strengths and magnetic field tunability, such that an efficient magnetic guiding of $\mathrm{H}$ atoms from zero up to the velocity of the initial supersonic beam $(\approx 500 \mathrm{~m} / \mathrm{s})$ can be achieved. The guide will act as a strong filter for carrier and precursor gas atoms and $\mathrm{H}$ atoms at other, unwanted velocities. As a magnetic counterpart to an electrostatic velocity filter, such a guide can also provide an access route to producing slow beams of paramagnetic atoms and molecules from supersonic or effusive beam sources.

\section{Magnetic field of a hexapole in Halbach configuration}

Hexapole magnets have been used in atomic physics since the 1950s. The first designs were based on six conducting wires with currents in alternating directions [28,29] or electromagnets with iron pole tips [30,31]. In the 1980s, multi-segment, permanent magnet multipole designs were developed to reduce chromatic aberrations affecting the experimental performance of a standard multipole magnet $[32,33]$. Magnetic hexapole focusing with these so-called Halbach arrays has been used in various experiments [34-41], and recently, even a magnetic deflector based on a Halbach configuration has been demonstrated [42].

The number of segments in a Halbach array is given by $S=m(2 n)$, where $n$ denotes the multipole order and $m \in$ $\mathbb{N}_{>0}$ [32]. For our hexapole setup $(n=3)$, we chose $m=2$ yielding a total number of $S=12$ segments. Figure 2 shows a schematic representation of such a Halbach array in hexapole configuration and a photograph of an array that has been built by Arnold Magnetic Technologies for our use.

The magnetic field of the Halbach array was modelled using the Radia 4.29 program [43-45] which allows for three-dimensional magnetostatics simulations, so that it was possible to estimate the influence of fringe fields at the end of the multipole magnets as compared to the two-dimensional, analytical treatment by Halbach $[32,33]$. The output was fitted to the multipole expansion of the 
magnetic field to allow for an easier implementation in the particle trajectory simulation program (Sect. 4).

In cylindrical coordinates, the multipole expansion of the magnetic field can be expressed as [46]

$$
\begin{aligned}
& B_{\phi}(r, \phi)=B_{0} \sum_{n=1}^{\infty}\left(b_{n} \cos (n \phi)+a_{n} \sin (n \phi)\right)\left(\frac{r}{r_{i}}\right)^{n-1} \\
& B_{r}(r, \phi)=B_{0} \sum_{n=1}^{\infty}\left(-a_{n} \cos (n \phi)+b_{n} \sin (n \phi)\right)\left(\frac{r}{r_{i}}\right)^{n-1},
\end{aligned}
$$

where $B_{0}$ is the remanence, $r_{i}$ is the inner radius of the multipole, $b_{n}$ are the "normal" multipole coefficients and $a_{n}$ are the "skew" coefficients. Owing to symmetry properties, the skew components in equations (2) and (3) vanish. Higher harmonics, $n^{\prime}$, are given by $n^{\prime}=n+\nu S$, where $\nu \in \mathbb{N}_{>0}$ [32]. In our case, it is sufficient to consider $n=3$ and the first higher multipole term $(\nu=1)$, and thus for $S=12$, we use $n^{\prime}=15$.

The results from Radia simulations (see below) suggest a significant contribution from edge effects requiring the introduction of an axial magnetic field component $B_{z}$ and a scaling factor $A(z)$. Following the reasoning by Ackermann and Weiland [47], the magnetic-field components can be written as follows:

$$
\begin{aligned}
& B_{\phi}(r, \phi, z)=A(z) B_{0} \sum_{n=3,15} b_{n} \cos (n \phi)\left(\frac{r}{r_{i}}\right)^{n-1} \\
& B_{r}(r, \phi, z)=A(z) B_{0} \sum_{n=3,15} b_{n} \sin (n \phi)\left(\frac{r}{r_{i}}\right)^{n-1} \\
& B_{z}(r, \phi, z)=\frac{d A(z)}{d z} B_{0} \sum_{n=3,15} b_{n} \sin (n \phi)\left(\frac{r^{n}}{n r_{i}^{n-1}}\right) .
\end{aligned}
$$

The normal multipole coefficients, $b_{3}$ and $b_{15}$, are found by fitting to the Radia simulation output in the centre of the hexapole (setting $A(z)=1$ ). For our chosen configuration with $B_{0}=0.7 \mathrm{~T}, r_{i}=3 \mathrm{~mm}, r_{o}=6 \mathrm{~mm}$ and $l_{h}=7.1 \mathrm{~mm}$, we obtain $b_{3}=0.86$ and $b_{15}=-0.11$.

The scaling factor $A(z)$ is obtained by fitting to a Glaser function [48] of the form

$$
A(z)=\frac{1}{\left(\left(1+(z / a)^{4}\right)^{2}\right)},
$$

where $a=d /(\sqrt{(\sqrt{2}-1)})$ is a "specific" length and $d$ is the full width at half maximum (FWHM) of the axial field distribution. The fit returned a value of $d=2.88 \mathrm{~mm}$.

Figure 3 illustrates that the output of the Radia simulations is in good agreement with the fit to equations (4), (5) and (7), thus justifying the use of a multipole expansion. The results obtained in the centre of the Halbach array are also consistent with the two-dimensional, analytical solution by Halbach [32] and with finite-element calculations (FEMM 4.2 [49], not shown) which provided a good cross-check on the Radia output values.

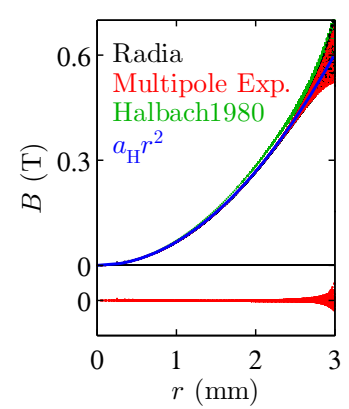

Fig. 3. Transverse magnetic field in the centre of a Halbach array (at all angles $\phi$ ). The output from Radia calculations is given by black dots. Results from a fit to the multipole expansion are represented by red dots. Residuals of the fit (in red colour) are vertically offset for clarity. The data are in close agreement with the two-dimensional, analytical solution to a Halbach array (green colour) [32] and they are wellapproximated by a quadratic dependency to $a_{\mathrm{H}} r^{2}$ (blue line; yielding $a_{\mathrm{H}}=0.07 \mathrm{~T} / \mathrm{mm}^{2}$ ), where $a_{\mathrm{H}} / 2$ is the magnetic field curvature.

\section{Hybrid magnetic guide}

In this paper, we present simulation results for a compact, bent hybrid guide $(R=180 \mathrm{~mm})$ which is based on the superposition of a magnetic hexapole field from a Halbach array and a second magnetic field shape generated using current-carrying wires. Two wire configurations are considered here: (a) a hexapole design tailored to match the shape of the Halbach hexapole field ('two-hexapole configuration') and (b) a configuration with two wire locations on opposite sides of the Halbach magnet ('deflection configuration'). The guide itself consists of six Halbach arrays (specifications as in Sect. 2) and $6 \times 4$ or $2 \times 4$ wires in the two-hexapole and deflection configuration; the geometry of the guide is illustrated in Figure 6b. The chosen geometry meets the requirements for the efficient, velocitytunable magnetic guiding of $\mathrm{H}$ atoms in low-field-seeking quantum states after Zeeman deceleration.

The magnetic field of the Halbach array is calculated using equations (4)-(6) and the magnetic field of each wire is approximated with the analytical solution of the BiotSavart law for a long straight current-carrying wire. In Cartesian coordinates, the magnetic field vector, $\boldsymbol{B}$, outside the wire is given by:

$$
\boldsymbol{B}=\left(\begin{array}{c}
B_{x} \\
B_{y} \\
B_{z}
\end{array}\right)=\frac{\mu_{0} I}{2 \pi R_{\mathrm{w}}^{2}}\left(\begin{array}{c}
-y \\
x \\
0
\end{array}\right)
$$

where $\mu_{0}$ is the permeability of free space, $I$ is the current through the wire and $R_{\mathrm{w}}$ is the distance to the centre of the wire. By adding the magnetic field components of different wires, which are mutually offset in the $x y$ plane, various wire configurations can be attained. Sets of four wires are chosen to match the dimensions of a Halbach array segment, and a wire diameter $d_{\text {wire }}=450 \mu \mathrm{m}$ was used. 

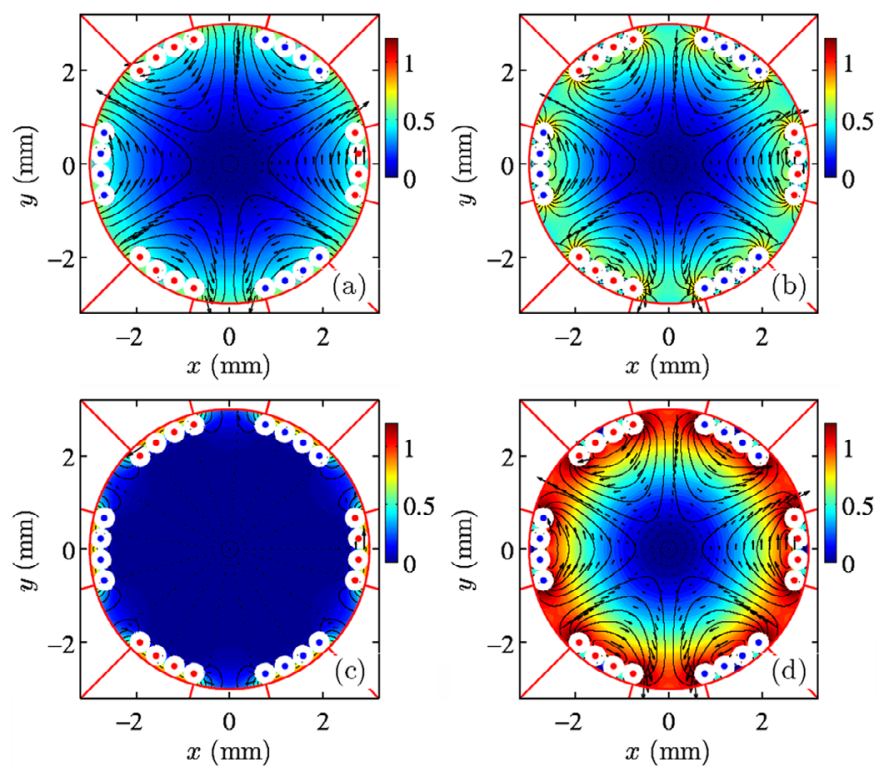

Fig. 4. Two-hexapole configuration: density plot of the magnetic field magnitude $B$ (in T) in the centre of a hybrid guide element and flux lines at different currents through the wires. The edges of the permanent magnet segments are indicated in red colour. Wires (pointing into the plane) are shown as filled white circles. Red (blue) dots indicate that the current is directed towards (out of) the plane of projection. The current is assigned with a positive (negative) value if the superposition with the Halbach magnetic field results in an increase (decrease) of the transverse magnetic field. (a) $I=0$ A, Halbach array only, (b) $I=500$ A, no Halbach array, (c) $I=-330$ A, (d) $I=500$ A.

\subsection{Two-hexapole configuration}

In the two-hexapole configuration (Fig. 4), six sets of four wires are arranged inside the Halbach array and operated at the same current but in alternating current directions. In this way, the wires generate a magnetic hexapole field whose extent matches with that of the Halbach magnets. Depending on the current direction, the application of a current then leads to an overall increase or decrease of the transverse magnetic field, and thereby changes the magnetic field gradient $d B(r) / d r$. In Figure 4, this increase (decrease) is illustrated at currents of $500 \mathrm{~A}(-330 \mathrm{~A})$. For $I=-330 \mathrm{~A}$, the opposing hexapole fields nearly cause a complete cancellation of the magnetic field magnitude in the transverse direction. Figure 5 shows that the resulting transverse magnetic field is well approximated by a quadratic dependency on the transverse position, i.e., $B(r)=a_{\mathrm{H}} r^{2}$. The parameter $a_{\mathrm{H}}$, a measure of the magnetic field curvature, changes linearly with the current applied to the wires (see inset).

Since the transverse magnetic field gradient for a magnetic hexapole depends on the off-axis particle position, i.e., $d B(r) / d r=2 a_{\mathrm{H}} r$, it is difficult to assess the maximum guidable velocity for such a guide using equation (1). An upper estimate of $v_{z, m}$ can be obtained by setting $r=r_{i}-d_{\text {wire }}$. The lower and upper limits for $a_{\mathrm{H}}$ correlate with wire currents of $-330 \mathrm{~A}$ and $500 \mathrm{~A}$ suggesting that

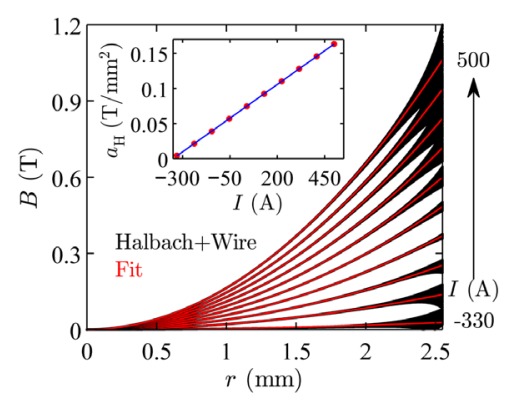

Fig. 5. Change of the transverse magnetic field inside a hybrid guide element (at all angles $\phi$ ) as a function of current, $I$, applied to the wires (two-hexapole configuration). Results from the analytical solution are shown in black colour; quadratic fits to $B(r)=a_{\mathrm{H}} r^{2}$ are indicated in red colour. The fitted $a_{\mathrm{H}}$ values are linearly dependent on the applied current (inset).
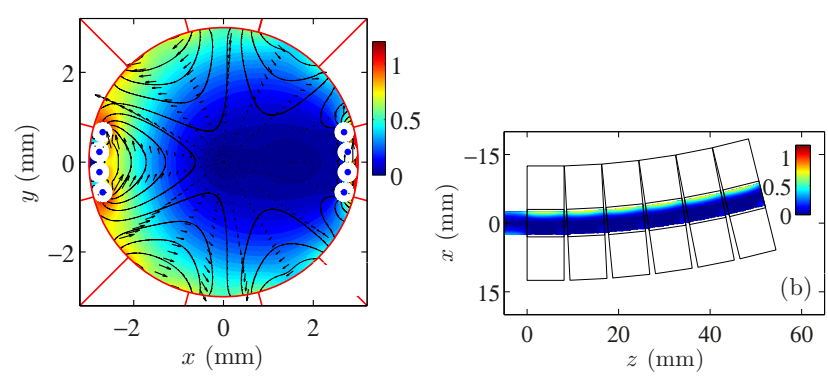

Fig. 6. (a) Density plot of the magnetic field magnitude $B$ (in $\mathrm{T})$ in the centre of a hybrid guide element for the deflection configuration including flux lines $(I=-400$ A.). The notation of the wires is the same as in Figure 4. (b) Cross-section view of $B$ inside a bent guide (at $y=0, R=180 \mathrm{~mm}$, to scale) with six guide sections using the configuration in (a). The position of the Halbach blocks is indicated to illustrate the physical arrangement. To achieve deflection in the opposite direction, the current direction through the wires is reversed.

the maximum guidable velocity for ground-state $\mathrm{H}$ atoms can be tuned between zero and roughly $900 \mathrm{~m} / \mathrm{s}$. Threedimensional particle trajectory simulations (Sect. 4) imply that this guess overestimates the true guidability for $\mathrm{H}$ atoms at different velocities.

\subsection{Deflection configuration}

In the deflection configuration (see Fig. 6), two series of four wires - carrying the same current in the same direction - are placed on opposite sides of the Halbach array. This leads to an increase of the magnetic field strength $B$ on the side of the Halbach magnets, where the flux lines of the Halbach magnets and the wires point in the same direction, while it causes a decrease in $B$ on the other side. Depending on the current direction, a magnetic deflection field in the $+x$ (Fig. 6) or $-x$ direction is created which either increases or counteracts the centrifugal force on the particles inside a bent guide. 


\section{Numerical particle trajectory simulations}

To simulate the guiding properties, three-dimensional numerical particle trajectory simulations were carried out [14]. To allow for variations of the guide structure (number of segments, bend radius etc.), the magnetic field components for the wires and the Halbach array are generated by rotation about the $y$-axis at the beginning of each simulation and stored as grid points. The influence of the exiting wires on the shape of the magnetic field was not considered. As the bend radius is large $(R=180 \mathrm{~mm})$, it is assumed that equation (8) is still a valid approximation for the magnetic vector field of the wires. The magnetic field is expressed in Cartesian coordinates requiring trilinear interpolation [50] for the evaluation of the field components and partial derivatives.

The program can propagate the positions and velocities of 100000 particles (in each Zeeman substate of ground-state atomic hydrogen) at a time through the guide, and it can also use existing trajectory data as input from a Zeeman-decelerator trajectory code. At each time step, particles are removed from the simulation if they have reached the walls of the guide (inner diameter of $5.1 \mathrm{~mm}$ ), given by the convex hull of points that mark the guide elements.

\section{Guiding efficiency}

\subsection{Continuous operation of the wire currents}

To study the guiding efficiency of $\mathrm{H}$ atoms under continuous application of a current to the wires, trajectory simulations were run at different currents for fixed initial longitudinal velocities $v_{z}\left(T_{z}=0\right)$. A set starting position in front of the guide $\left(z_{0}=-2 \mathrm{~mm}\right.$ for all particles $)$ and a transverse temperature of $10 \mathrm{mK}$ centred around $v_{r}=0$ are used (cf. [14]). A uniform transverse spread over the inner diameter of a guide element is assumed.

From the simulation results, the transmission of $\mathrm{H}$ atoms in the two low-field-seeking quantum states is obtained and shown in Figure 7 as a function of initial longitudinal velocity and wire current for both the twohexapole configuration and the deflection configuration. The number of transmitted particles without a magnetic moment $(<3 \%)$ and $\mathrm{H}$ atoms in high-field-seeking states $(<8 \%)$ is negligible over the whole parameter range studied (not shown).

Figure 7 confirms that the maximum guidable velocity increases as the current through the wires is increased. The trends are similar for both wire configurations, with an advantage of the deflection configuration in terms of maximum transmission at different velocities. In both cases, at a given current, there is a gradual increase in guidability as a function of initial $v_{z}$ which is due to the transverse distribution of positions and velocities inside the guide. For example, at a current of $200 \mathrm{~A}$ in the deflection configuration, the transmission is about $90 \%$ for low-field-seeking particles with $v_{z}=250 \mathrm{~m} / \mathrm{s}$ and $50 \%$ for particles with $v_{z}=400 \mathrm{~m} / \mathrm{s}$; and the guidability falls below $10 \%$ above
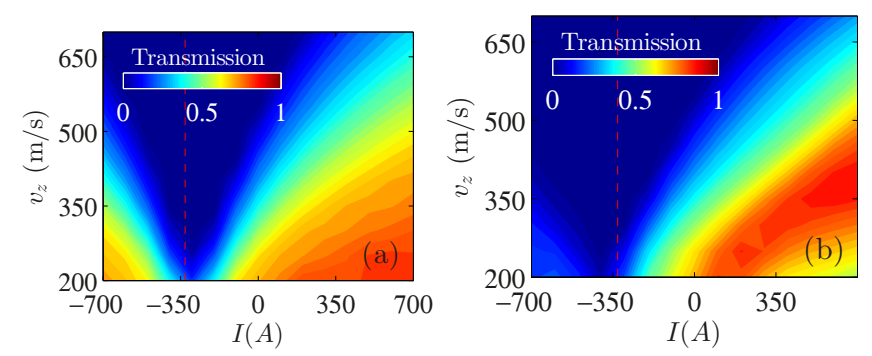

Fig. 7. Density plot showing the transmission of $\mathrm{H}$ atoms in the two low-field-seeking quantum states as a function of initial longitudinal velocity, $v_{z}$ and current applied to the wires, $I$. The guidability is given on a scale from 0 (no transmission) to 1 (all particles transmitted). (a) Two-hexapole configuration; (b) deflection configuration. The red dashed line denotes the current $I_{1}$ that is used for the simulation of the guiding efficiency in pulsed mode (see Sect. 5.2).

a velocity of $600 \mathrm{~m} / \mathrm{s}$. This implies that a clear separation of similar particle velocities is difficult to achieve in a continuous mode of operation, and efforts to guide only the low-velocity tail would almost certainly result in a low particle transmission efficiency. A similar behaviour is expected for a guide setup in which the guided velocities are adjusted through a change in bend radius.

\subsection{Pulsed operation}

As can be seen from Figure $7 \mathrm{~b}$, magnetic guiding at $-400 \mathrm{~A}$ in the deflection configuration is inefficient for all but very low particle velocities $(<200 \mathrm{~m} / \mathrm{s})$. On the other hand, the transmission through the guide is predicted to be as high as $80 \%$ above $0 \mathrm{~A}$, and the initial longitudinal velocity with the highest efficiency transmission gradually increases towards higher currents. Since the decelerated packet of $\mathrm{H}$ atoms is temporally offset from the other particles in the supersonic beam, operation of the guide in switched mode at two currents, $I_{1}$ and $I_{2}$, may significantly improve the velocity selection of the guide.

For this scheme, the guide is switched to a current $I_{1}$, i.e., $I_{1}=-330 \mathrm{~A}$ for the two-hexapole configuration and $I_{1}=-400 \mathrm{~A}$ for the deflection configuration, at time $t_{1}$. Here, $t_{1}$ is defined as the arrival time of the first $\mathrm{H}$ atoms from the supersonic beam. The duration of current pulse $1, \Delta t_{I_{1}}$, is scanned to find the optimum settings between efficient guidability for the decelerated particles and low transmission for particles at other velocities. The current is then switched to $I_{2}$, which is the lowest current required to achieve a high transmission of the decelerated beam. In this way, higher velocities are guided less efficiently and ohmic heating of the wires is minimised. The duration of the second current pulse, $\Delta t_{I_{2}}$, is determined by the time-of-flight of the decelerated particles through the guide. Owing to the low inductance of the short wires ( $\approx 60 \mathrm{~mm}$ ), switching between the two currents should be almost instantaneous.

Operation of the guide in pulsed mode was simulated for a Zeeman-decelerated supersonic beam of $\mathrm{H}$ atoms 


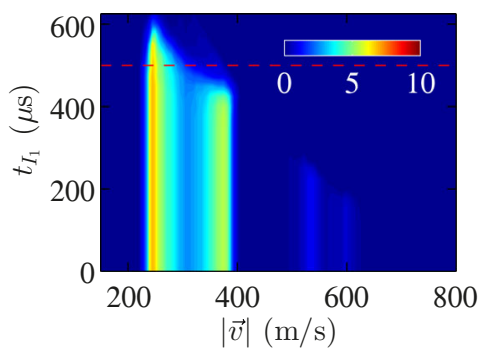

Fig. 8. Density plot showing the relative transmission of Zeeman decelerated and guided $\mathrm{H}$ atom velocities $|\boldsymbol{v}|$ (low-fieldseeking states only), using a deceleration pulse sequence to produce a beam at $240 \mathrm{~m} / \mathrm{s}$, as a function of pulse duration $\Delta t_{I_{1}}$ in the deflection configuration; arbitrary scaling. The red dashed line highlights the velocity distribution at $\Delta t_{I_{1}}=500 \mu$ s used to determine the results in Figure 9.

whose properties were closely matched to experimental conditions [14]. The entrance of the guide was set at $2 \mathrm{~mm}$ behind the detection laser position used in experiments (about $40 \mathrm{~mm}$ behind the last deceleration coil). A deceleration pulse sequence was chosen such that the $\mathrm{H}$ atom beam velocity was decreased from $500 \mathrm{~m} / \mathrm{s}$ to $240 \mathrm{~m} / \mathrm{s}$ using 11 deceleration coils at $243 \mathrm{~A}$ (coil 6 used as a bias coil at $\left.-30 \mathrm{~A}, \kappa_{0}=0\right)$. Figure $9 \mathrm{c}$ illustrates that there is good agreement between the experimental (vertically offset) and simulated time-of-flight traces for Zeeman deceleration. Note that, in the experimental data, all $\mathrm{H}$ atoms in both low- and high-field-seeking states (covered by the detection laser volume) are displayed. In contrast to that, low- and high-field-seekers (all particles in the $x y$ detection plane) are shown separately in the simulated traces.

The simulation results for guiding in the deflection configuration are shown in Figure 8 using $I_{1}=-400 \mathrm{~A}$ and $I_{2}=150 \mathrm{~A} ; t_{1}=300 \mu \mathrm{s}$ after $\mathrm{H}$ atom production. Similar characteristics are observed for the two-hexapole configuration, but - as expected from Figure 7 - the guiding efficiency for the decelerated beam is lower.

Figure 8 shows a density plot of the transmission efficiency for the guided $\mathrm{H}$ atom velocities (low-field-seeking states only) as a function of the pulse duration $\Delta t_{I_{1}}$. Here, $\Delta t_{I_{1}}=0 \mu \mathrm{s}$ corresponds to the case where $I_{2}=150 \mathrm{~A}$ at all times. The overall transmission through the guide decreases the longer the pulse duration $\Delta t_{I_{1}}$, which is expected at this current. Even for $\Delta t_{I_{1}}=0 \mu \mathrm{s}$, the guiding efficiency for particle velocities above $400 \mathrm{~m} / \mathrm{s}$ is low. However, under these conditions, not only the decelerated bunch of particles at $240 \mathrm{~m} / \mathrm{s}$, but also a packet moving at a velocity of $350 \mathrm{~m} / \mathrm{s}$ is transmitted ( $50 \%$ of the intensity). By increasing $\Delta t_{I_{1}}$ to $500 \mu \mathrm{s}$, the guidability for this second packet is widely reduced, while the decelerated beam at a velocity of $240 \mathrm{~m} / \mathrm{s}$ is still guided at high efficiency $(\approx 70 \%)$.

In Figures 9a and 9b, the initial velocity distribution after Zeeman deceleration and the velocity distribution after subsequent guiding are compared. The excellent discrimination against high velocities and efficient transmission of the decelerated peak is clear from the fig-
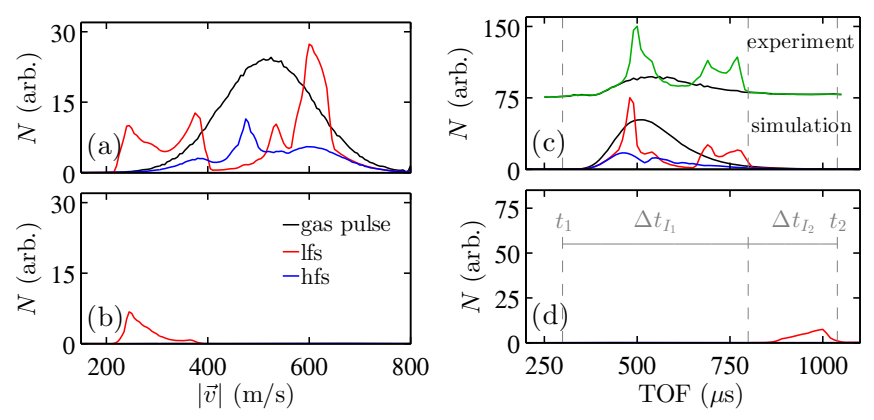

Fig. 9. (a) Simulated velocity distribution of $\mathrm{H}$ atoms after Zeeman deceleration and (b) after magnetic guiding in the deflection configuration $\left(\Delta t_{I_{1}}=450 \mu \mathrm{s}, \Delta t_{I_{2}}=250 \mu \mathrm{s}\right)$ following Zeeman deceleration; same scaling as in Figure 8. In the legend, 'lfs' ('hfs') denotes particles in low (high)-field-seeking quantum states and 'gas pulse' stands for particles whose motion is not influenced by a magnetic field. (c) and (d) Time-of-flight profiles for the same output data as in (a) and (b). Experimental traces (all particles within the detection laser volume, see text for details) are vertically offset for clarity. In the (black) green curve, the magnetic fields of the deceleration coils are (not) switched.

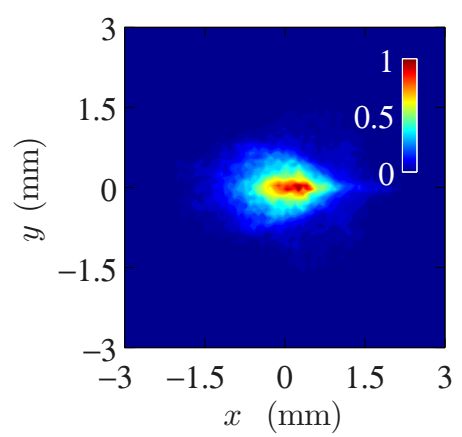

Fig. 10. Density plot of the simulated transverse particle distribution of $\mathrm{H}$ atoms (at the guide exit; low-field-seeking states only) after Zeeman deceleration and magnetic guiding. The distribution is normalised and shown in the frame of reference following the bend of the guide. Same settings as in Figure 9.

ure. Furthermore, none of the carrier gas particles would be transmitted, and the guiding of particles in high-fieldseeking states is several orders of magnitude smaller than for the decelerated particles.

Due to the compact design of the guide, the flight time of the decelerated $\mathrm{H}$ atoms through the guide still remains relatively short (here, $250 \mu \mathrm{s}$ ). Nevertheless, as shown in Figure 9d, the peak intensity of the time-of-flight signal decreases by about a factor of three owing to the lack of focusing along the beam axis inside the guide. For the study of cold-ion molecule reactions, the longitudinal beam spread is not an issue, because only the overall flux of decelerated particles through the ion trap matters. For other applications, a combination of the guide with a bunching scheme may be beneficial.

Figure 10 shows that the transverse distribution of the exiting, slow $\mathrm{H}$ atom beam (low-field-seeking states only) is centred along the axis of the magnetic guide, with a 
half-width of about $0.5 \mathrm{~mm}$ in either direction. Hence, in this case, the transverse extent of the guided beam is very well matched to the size of a typical Coulomb crystal in an ion trap ( $\sim$ few hundred $\mu \mathrm{m})$. The use of several magnetic hexapoles as guiding elements renders the thin-lens approximation invalid, such that there is no well-defined focal point behind the guide. Owing to the strong transverse forces inside the guide, the exiting beam will rapidly diverge in the transverse direction. This effect will be most prominent at very low particle velocities and provisions must be made to minimise the spreading, e.g. through further transverse focusing and/or by placing the ion trap as close to the guide exit as possible. Likewise, the transverse beam spread will be smallest if the guide is directly attached to the Zeeman decelerator.

Further work is required to assess the transverse distribution of the guided particles for different final velocities after Zeeman deceleration, since the position of the exiting particles depends strongly on the switching times for the guide, the applied current and the longitudinal beam velocity. Owing to the higher centrifugal force, faster particles are horizontally offset with respect to the slower ones, and it is not clear whether this effect can be compensated for solely by changing the applied guiding current. If there is a significant transverse offset, other means need to be found to match the guided beam to the position of the Coulomb crystal, e.g. via mechanical alignment or through a re-direction of the beam. With regard to an optimisation of the guiding scheme, it would also be interesting to look at different relative transverse positions (in the $x$ direction) between the Zeeman decelerator and the magnetic guide, as this will influence particle transmission. Even though the length of the guide is very short compared to the bend radius, other transverse dynamics, similar to those observed in molecular synchrotrons [51], may also have to be taken into account.

\section{Other applications}

The proposed guide configurations could also be used for the magnetic velocity selection of effusive or supersonic beams, similar to bent magnetic guides developed previously $[21,24,25]$. Such a bent guide would form the magnetic counterpart to the electric velocity filter developed by the Rempe group [52], i.e., it would reject all but the slowest paramagnetic particles from a given velocity distribution. In Figure 11 the result of a simulation is shown for a $500 \mathrm{~m} / \mathrm{s}$ supersonic beam, directed into the guide behind a $2 \mathrm{~mm}$ diameter skimmer. The guide is operated at currents ranging from $-400 \mathrm{~A}$ to $700 \mathrm{~A}$, using either the twohexapole or the deflection configuration. Here, the guide is used in a continuous mode, but in experiments, pulsing for the time-of-flight of a supersonic beam $(\approx 200 \mu \mathrm{s})$ would be sufficient.

Figure 11 shows that this scheme can indeed be used to selectively guide particles from the low-velocity tail of the initial particle velocity distribution. At zero current through the wires, the simulation predicts that the maximum of the velocity distribution is shifted to $350 \mathrm{~m} / \mathrm{s}$,
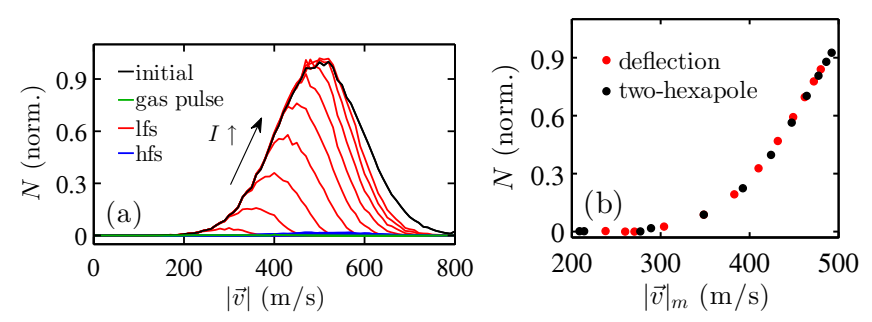

Fig. 11. (a) Simulated velocity distributions after magnetic velocity selection in the deflection configuration at different wire currents assuming an undecelerated supersonic beam of $\mathrm{H}$ atoms with an initial velocity distribution centered at $500 \mathrm{~m} / \mathrm{s}$. Curves are separately given for non-magnetic particles (green curve, always zero) and particles in low-field-seeking (lfs, red curves) and high-field-seeking quantum states (hfs, blue curves). The initial velocity distribution is shown for comparison (black curve). In the legend, 'lfs' ('hfs') denotes particles in low (high)-field-seeking quantum states, 'gas pulse' stands for particles whose motion is not influenced by a magnetic field and 'initial' marks the initial velocity distribution. (b) Normalised number of transmitted $\mathrm{H}$ atoms in low-fieldseeking states as a function of most probable velocity, $|\boldsymbol{v}|_{m}$, after magnetic guiding of a $500 \mathrm{~m} / \mathrm{s}$ supersonic beam of $\mathrm{H}$ atoms in the two-hexapole (black dots) and deflection configuration (red dots).

with $10 \%$ of the particles in low-field-seeking quantum states being transmitted. Depending on the current through the guide, the maximum of the velocity distribution can then be shifted to higher values (currents with a positive sign) or lower values (currents with a negative sign). In addition to that, the number of transmitted carrier gas particles and particles in high-field-seeking states is several orders of magnitude lower than the initial number of particles, thus ensuring high quantum-state selectivity. As shown in Figure 11b, the number of particles drops off towards lower velocities, even though most particles at these velocities are being guided. The Figure also illustrates that the number of guided particles is very similar for the two-hexapole and the deflection configuration, but different currents are required to achieve the same velocity distribution (cf. Sect. 5.1).

The above example illustrates that even undecelerated supersonic beams of $\mathrm{H}$ atoms can be magnetically guided, and this mode of operation may prove useful for the initial testing of such a hybrid guide. Likewise, a guide in this configuration would allow for preliminary collisionenergy dependent ion-atom collision experiments, without the additional complexity of a Zeeman decelerator. Using different guide geometries (higher remanence for the Halbach segments, lower bend radii, more guide elements), the velocity-dependent guiding of numerous other paramagnetic species would be feasible. The guide could also be used in combination with buffer-gas-cooled sources, similar to pulsed electric guides [53]. If operated in a pulsed mode, such a guide also presents an alternative way to produce a quantum-state-selected supersonic beam with a narrow kinetic energy distribution. 


\section{Conclusions}

The particle trajectory simulations with $\mathrm{H}$ atoms presented here demonstrate that a bent hybrid magnet design can provide a very efficient means to guide a selected element of the particle velocity distribution, particularly in the switched mode of operation. In addition to that, it will ensure a high degree of quantum-state selection and a nearly complete removal of residual carrier gas, which is particularly important for a guide at the end of a short Zeeman decelerator such as in our laboratory.

The deflection configuration, when used in the pulsed mode of operation, gives the best discrimination between decelerated atoms and other atoms and is also likely to be easier to set up than the two-hexapole configuration, i.e., less ohmic heating and lower mechanical complexity with regard to misalignment between the wires and the Halbach array segments. In the case of a two-hexapole guide, the proposed guide increases the accessible range of transverse magnetic field gradients by a factor of two in comparison to a guide design made solely from current-carrying wires and operated at the same currents. In both deflection and two-hexapole cases, the guiding of a certain velocity range can be achieved without the application of a current through the wires, which would also be useful for the initial characterisation of such an experimental setup. As the guidable velocity range is determined by the remanence of the permanent magnet material and the bend radius, the geometry of a bent hydrid magnetic guide could be tailored to match the requirements for the velocity-selective guiding of a large number of different paramagnetic atoms and molecules.

This work is financed by the Engineering and Physical Sciences Research Council (U.K.) EPSRC(GB) under Projects Nos. EP/G00224X/1 and EP/1029109.

\section{References}

1. R.V. Krems, Phys. Chem. Chem. Phys. 10, 4079 (2008)

2. G. Quéméner, P.S. Julienne, Chem. Rev. 112, 4949 (2012)

3. S. Ospelkaus, K.K. Ni, D. Wang, M.H.G.D. Miranda, B. Neyenhuis, G. Quéméner, P.S. Julienne, J.L. Bohn, D.S. Jin, J. Ye, Science 327, 853 (2010)

4. A.B. Henson, S. Gersten, Y. Shagam, J. Narevicius, E. Narevicius, Science 338, 234 (2012)

5. S. Chefdeville, Y. Kalugina, S.Y.T. van de Meerakker, C. Naulin, F. Lique, M. Costes, Science 341, 1094 (2013)

6. J. Jankunas, K. Jachymski, M. Hapka, A. Osterwalder, J. Chem. Phys. 142, 164305 (2015)

7. S.Y.T. van de Meerakker, H.L. Bethlem, N. Vanhaecke, G. Meijer, Chem. Rev. 112, 4828 (2012)

8. J. Jankunas, A. Osterwalder, Annu. Rev. Phys. Chem. 66, 241 (2015)

9. I.W.M. Smith, Annu. Rev. Astron. Astr. 49, 29 (2011)

10. M.T. Bell, Ph.D. thesis, University of Oxford, 2008

11. B.R. Heazlewood, T.P. Softley, Annu. Rev. Phys. Chem. 66, 475 (2015)

12. J. Lam, C.J. Rennick, T.P. Softley, Rev. Sci. Instrum. 86, 053108 (2015)
13. S.D. Hogan, A.W. Wiederkehr, M. Andrist, H. Schmutz, F. Merkt, J. Phys. B 41, 081005 (2008)

14. K. Dulitz, M. Motsch, N. Vanhaecke, T.P. Softley, J. Chem. Phys. 140, 104201 (2014)

15. K.J. Kügler, K. Moritz, W. Paul, U. Trinks, Nucl. Instrum. Methods 228, 240 (1985)

16. D. Thompson, R.V.E. Lovelace, D.M. Lee, J. Opt. Soc. Am. B 6, 2227 (1989)

17. R. Folman, P. Krüger, J. Schmiedmayer, J. Denschlag, C. Henkel, in Advances In Atomic, Molecular, and Optical Physics, edited by B. Bederson, H. Walther (Academic Press, 2002), Vol. 48, pp. 263-356

18. J. Fortágh, C. Zimmermann, Rev. Mod. Phys. 79, 235 (2007)

19. A. Tonyushkin, M. Prentiss, J. Appl. Phys. 108, 094904 (5) (2010)

20. M. Greiner, I. Bloch, T.W. Hänsch, T. Esslinger, Phys. Rev. A 63, 031401 (2001)

21. B. Ghaffari, J.M. Gerton, W.I. McAlexander, K.E. Strecker, D.M. Homan, R.G. Hulet, Phys. Rev. A 60, 3878 (1999)

22. R.G. Hulet, K. Strecker, J. Gerton, W. McAlexander, C. Bradley, J. Tollett, C. Sackett, B. Ghaffari, S. Moss, Method and apparatus for magnetically guiding neutral particles (US Patent 6657188B1, 2003)

23. D. Patterson, J.M. Doyle, J. Chem. Phys. 126, 154307 (2007)

24. A. Goepfert, F. Lison, R. Schütze, R. Wynands, D. Haubrich, D. Meschede, Appl. Phys. B 69, 217 (1999)

25. J.P. Beardmore, A.J. Palmer, K.C. Kuiper, R.T. Sang, Rev. Sci. Instrum. 80, 073105 (2009)

26. B. Bertsche, J. Jankunas, A. Osterwalder, Chimia 68, 256 (2013)

27. A.P.P. van der Poel, K. Dulitz, T.P. Softley, H.L. Bethlem, New J. Phys. 17, 055012 (2015)

28. H. Friedburg, Z. Phys. A 130, 493 (1951)

29. H. Friedburg, W. Paul, Naturwissenschaften 38, 159 (1951)

30. A. Lemonick, F.M. Pipkin, Phys. Rev. 95, 1356 (1954)

31. A. Lemonick, F.M. Pipkin, D.R. Hamilton, Rev. Sci. Instrum. 26, 1112 (1955)

32. K. Halbach, Nucl. Instrum. Methods 169, 1 (1980)

33. K. Halbach, Nucl. Instrum. Methods 187, 109 (1981)

34. P. Schiemenz, A. Ross, G. Graw, Nucl. Instrum. Methods A 305, 15 (1991)

35. W.G. Kaenders, F. Lison, A. Richter, R. Wynands, D. Meschede, Nature 375, 214 (1995)

36. W.G. Kaenders, F. Lison, I. Müller, A. Richter, R. Wynands, D. Meschede, Phys. Rev. A 54, 5067 (1996)

37. D. Szczerba, L.D. van Buuren, J.F.J. van den Brand, H.J. Bulten, M. Ferro-Luzzi, S. Klous, H. Kolster, J. Lang, F. Mul, H.R. Poolman, M.C. Simani, Nucl. Instrum. Methods A 455, 769 (2000)

38. S. Dworski, G. Alexandrowicz, P. Fouquet, A.P. Jardine, W. Allison, J. Ellis, Rev. Sci. Instrum. 75, 1963 (2004)

39. T. Oku, S. Yamada, H. Sasao, J.I. Suzuki, T. Shinohara, K. Hirota, K. Ikeda, T. Tsuzaki, Y. Kiyanagi, M. Furusaka, H.M. Shimizu, Physica B 385, 1225 (2006)

40. R. Chaustowski, V. Leung, K. Baldwin, Appl. Phys. B 86, 491 (2007)

41. L.T. Sun, H.W. Zhao, Z.M. Zhang, H. Wang, B.H. Ma, X.Z. Zhang, X.X. Li, Y.C. Feng, J.Y. Li, X.H. Guo, Y. Shang, H.Y. Zhao, Rev. Sci. Instrum. 78, 053302 (2007) 
42. J. Gardner, R. Castillo-Garza, M.G. Raizen, J. Chem. Phys. 139, 096103 (2013)

43. J. Chavanne, Radia (version 4.29), http://www.esrf. eu/Accelerators/Groups/InsertionDevices/Software/ Radia [2014, Aug 2] (2009)

44. P. Elleaume, O. Chubar, J. Chavanne, Proceedings of the 1997 Particle Accelerator Conference (PAC-97), 12-16 May 1997 (Vancouver, BC), Vol. 3, p. 3509

45. O. Chubar, P. Elleaume, J. Chavanne, J. Synchrotron Radiat. 5, 481 (1998)

46. J. Rossbach, P. Schmüser, Conf. Proc. C9209071, 17 (1993)

47. W. Ackermann, T. Weiland, Proceedings of the 2005 Particle Accelerator Conference (PAC 05), 16-20 May 2005, Knoxville, Tennessee, p. 4036

48. W. Glaser, Z. Phys. A - Hadron Nucl. 117, 285 (1941)

49. D.C. Meeker, Finite element method magnetics (version 4.2), http://www.femm.info [2014, Aug 2] (2010)
50. H.R. Kang, in Computational Color Technology (SPIE Press, Bellingham, Washington USA, 2006), Chap. 9, pp. 151-159

51. C.E. Heiner, G. Meijer, H.L. Bethlem, Phys. Rev. A 78, $030702(2008)$

52. S.A. Rangwala, T. Junglen, T. Rieger, P.W.H. Pinkse, G. Rempe, Phys. Rev. A 67, 043406 (2003)

53. C. Sommer, M. Motsch, S. Chervenkov, L.D. van Buuren, M. Zeppenfeld, P.W.H. Pinkse, G. Rempe, Phys. Rev. A 82, $013410(2010)$

Open Access This is an open access article distributed under the terms of the Creative Commons Attribution License (http://creativecommons.org/licenses/by/4.0), which permits unrestricted use, distribution, and reproduction in any medium, provided the original work is properly cited. 